\title{
Verbal learning and reinforcement: A reexamination of the Premack hypothesis*
}

\author{
ROBERT W. SCHAEFFER and ROBERT J. NOLAN \\ Auburn University, Auburn, Alabama 36830
}

\begin{abstract}
This study replicates a previous study which purportedly tested and confirmed the applicability of the Premack principle to verbal learning. Ninety male and female Ss learned paired associate (PA) lists (comprised of words of intermediate response probabilities) in which the response words were reinforced (i.e., immediately followed) by words of high, intermediate, or low probabilities. Word probabilities were obtained from two standard word count sources. As was anticipated, no differential effects of the probability of the "reinforcement" words were obtained, as measured by trials to criterion for learning the PA lists. The failure of this experiment to support this application of the Premack principle was attributed to inappropriate methodologies used in identifying word probabilities. An appropriate method for testing the Premack principle in a PA learning task was described.
\end{abstract}

The Premack principle $(1959,1965)$ conceptualizes reinforcement as a relative relation between differentially probable responses in which more probable responses will reinforce less probable responses. In an early attempt to apply Premack's conception of reinforcement to verbal learning, McIntire (1963) made the assumption that the frequency of occurrence of a word, as measured by a word count source such as Thorndike and Lorge (1944), would be highly correlated with the response probability of that word in an individual S's speech. If one grants the plausibility of this assumption, then words of a high frequency of occurrence, as measured by the Thorndike-Lorge word list, should be capable of reinforcing the acquisition of words of lesser probabilities. This, according to McIntire, was the consequence that he expected from the Premack principle.

Although McIntire's experiment produced data which he interpreted as supportive of the Premack principle, the present authors had several reasons to question the appropriateness of this technique for assessing the validity of the Premack principle. First, it does not seem plausible to assume that the frequency of occurrence of words presented in written form in literary sources in 1944 would necessarily be correlated in a highly positive manner with an individual S's written or oral word usage in 1944, let alone in 1963. Second, the Premack principle requires that the probabilities of all responses be determined for the individual $S$ in a situation in which $\mathrm{S}$ is able to choose freely between concurrently available responses. The principle does not permit response probability approximations by some assumed to be equivalent, or experimenter designated, surrogate response measures. In this context, the Thorndike-Lorge word list clearly fails to provide a direct measure of the

\footnotetext{
*Appreciation is due to Mary V. Moore, June Covin, and
} Bryan Crow for their assistance in data collection. response probability measures required by the Premack principle. Third, procedural difficulties, including words used by McIntire that cannot be located in the Thorndike and Lorge source, and word frequencies reported by McIntire that do not agree with the frequencies reported by Thorndike and Lorge, caused us to further question the generalizability of McIntire's data. Consequently, the present study, which was undertaken as a direct replication of McIntire's study, retested the hypothesis that the learning of a paired associate list can be facilitated when the second word of each pair is followed by a more probable third word.

\section{METHOD}

\section{Learning Materials}

Six lists of 10 paired associates (PA) were constructed. Three lists consisted of four-letter words derived from the Thorndike and Lorge (1944) word count, and three lists consisted of four-letter words derived from the Kucera and Francis (1967) word count. These lists are presented in Table 1. Lists of high-, medium-, and low-occurrence three-letter words were also derived from each of the two word count sources mentioned above. These three-letter words, which were used as reinforcement words, are presented in Table 2 . The combination of each PA list with each type of reinforcement word (derived from the same sources as the PA lists) thus provided 18 different sets of learning materials.

\section{Subjects}

All 90 Ss (47 males and 43 females) were volunteer Ss who were enrolled in introductory psychology classes at Auburn University. There were $5 \mathrm{Ss}$ in each of the 18 groups, corresponding to the 18 sets of learning materials used. Following completion of the experiment, debriefing sessions were held to inform Ss of the purpose and results of the experiments.

\section{Procedure}

The experiment employed: (a) a set of index cards, which was utilized during preliminary training and (b) two LaFayette memory drums, each with four presentation windows, which were utilized in the experiment proper. Preliminary training, using the index cards, was undertaken to insure each S's ability 
Table 1

Paired-Associate Lists*

\begin{tabular}{|c|c|c|c|c|c|}
\hline $\begin{array}{l}\text { Thorndike and Lorge } \\
\text { List I† }\end{array}$ & $\begin{array}{l}\text { Word Source } \\
\text { List II }\end{array}$ & $\begin{array}{c}\text { (Stimulus-Response) } \\
\text { List III }\end{array}$ & $\begin{array}{l}\text { Kucera and Francis } \\
\text { List IV }\end{array}$ & $\begin{array}{l}\text { Word Source } \\
\text { List V }\end{array}$ & $\begin{array}{c}\text { (Stimulus-Response) } \\
\text { List VI }\end{array}$ \\
\hline BELT-SEAL & FADE-STEM & BEAM-LUCK & TOUR-VICE & POEM-SELL & RING-SOUL \\
\hline BARN-RAGE & FOND-LIMB & MATE-CURE & PINK-RIDE & ASIA-IRON & CUBA-PATH \\
\hline FILE-BATH & HOOK-PEER & NAVY-BEND & FILL-MILE & HOLY-HANS & LIES-SELF \\
\hline DOLL-BEAN & BOLD-WEEP & TRIM-DUCK & EASE-SOLD & COAT-WAVE & BARS-SEED \\
\hline CASH-LAMB & WRAP-RARE & WIPE-ECHO & MAMA-RODE & TEND-SALE & DENY-PAIR \\
\hline CURL-HOST & BIND-QUIT & HEAP-TOSS & POPE-COOK & SALT-MILK & TILL-MEAT \\
\hline SHED-CREW & SORE-RICE & SNAP-IDLE & SUIT-BAND & MERE-CAST & MAIL-INCH \\
\hline RISK-CLAY & GOWN-BLEW & KICK-DULL & PACE-DEAN & GUNS-LOAN & DIRT-CODE \\
\hline TIDE-FUND & SANG-HAIL & TRAP-WHIP & LOTS-TINY & SHUT-RARE & LOAD-HATE \\
\hline HILL-DRUM & VINE-HATH & TOOL-FORT & SKIN-ADAM & MOLD-PIKE & WIRE-SAKE \\
\hline
\end{tabular}

*All words occur with a frequency between 38 and 50 times per million, as indicated in the respective sources, with the one exception of the word "HILL" in List I.

tList I is taken directly from McIntire (1963); the word "HILL" actually occurs with a frequency greater than 100 times per million, according to Thorndike and Lorge.

to give appropriate responses to the standard word anticipation method used in PA learning experiments (e.g., Kausler, McLaughlin, \& Kulik, 1962; Hawker, 1964). Preliminary training with the index cards produced the following response pattern from each S: (a) the $S$ read aloud the four-letter stimulus word when it was presented; (b) then the $S$ anticipated or guessed the four-letter response word prior to its appcarance (c) independently of whether S had or had not correctly guessed the four-letter response word, the $S$ read aloud the response word when it appeared and, (d) finally, the $S$ read aloud the three-letter reinforcement word. As per instructions, S did not try to guess the three-letter word, but merely read it aloud when it was presented. This response pattern occurred for each combination of a reinforcement word and a PA word pair. None of the words that appeared on the index cards that were utilized in preliminary training were used in the actual experiment.

When S had completed one errorless trial on the PA list used on the index cards, essentially the same instructions employed by McIntire (1963) then were used to acquaint $\mathrm{S}$ with the appropriate responses to be given to the stimulus, response, and reinforcement words as they were presented by the memory drum apparatus. As was the case with the index card procedure, the standard anticipation method for the learning of PA lists was used with the memory drums.

Each S was presented with 10 pairs of four-letter words on the memory drums. A four-letter stimulus word was presented for $2 \mathrm{sec}$, then the paper moved up to expose the associated four-letter response word; $2 \mathrm{sec}$ later, the paper moved up to expose a three-letter reinforcement word. Thus, the following response pattern was obtained from each S: (a) when the four-letter stimulus word was presented, $S$ read it aloud (b) immediately, the $\mathrm{S}$ guessed the four-letter response word (c) independently of whether $S$ had or had not identified correctly the response word, S read aloud the response word when it appeared (d) $S$ then read the three-letter reinforcement word aloud when it appeared, but did not try to guess the three-letter reinforcement word prior to its appearance.

The procedures described above were continued with each $\mathrm{S}$ until $S$ had successfully anticipated, without error, all 10 four-letter response words on the specific PA list $S$ was required to learn. Number of trials to this learning criterion served as the dependent variable in this experiment.

\section{RESULTS AND DISCUSSION}

The trials to criterion scores were analyzed for the Thorndike and Lorge PA lists using a simple two-way a nalysis of variance. This analysis revealed no statistically significant difference between reinforcement conditions (high, medium, and low reinforcement words $)(\mathrm{F}=.995 ; \mathrm{df}=2 / 36)$, no statistically significant difference between $P A$ lists $(F=.673 ; \mathrm{df}=2 / 35)$, and no statistically significant interaction of these factors $(F=.943 ; \mathrm{df}=4 / 36)$ at the .05 probability level. A two-way analysis of variance of the trials to criterion scores for the Kucera and Francis PA lists revealed no statistically significant difference between reinforcement conditions $(\mathrm{F}=3.17 ; \quad \mathrm{df}=2 / 36)$, no statistically significant difference between PA lists $(\mathrm{F}=.816$; $\mathrm{df}=$ $2 / 36$ ), and no statistically significant interaction of these factors $(F=.576 ; \mathrm{df}=4 / 36)$ at the .05 probability level.

Table 2

Reinforcement Words*

\begin{tabular}{|c|c|c|c|c|c|}
\hline \multicolumn{3}{|c|}{$\begin{array}{c}\text { Thorndike and Lorge } \\
\text { Word Source } \\
\text { Frequency }\end{array}$} & \multicolumn{3}{|c|}{$\begin{array}{l}\text { Kucera and Francis } \\
\text { Word Source } \\
\text { Frequency }\end{array}$} \\
\hline High $\dagger$ & Medium & Low & High & Medium & Low \\
\hline AGE & FED & ADO & SUN & CRY & $\mathrm{APE}$ \\
\hline END & DAD & $\mathrm{COB}$ & HIT & VIA & DEW \\
\hline HIM & HEN & DOL $† \dagger$ & EYE & CUP & ELM \\
\hline NOT & TON & DUN & LOT & ICE & HOG \\
\hline OLD & JAR & EFT & ASK & SUM & LAG \\
\hline OUR & PIG & FAD & HOT & FUN & MOP \\
\hline RAN & ROB & HIE & MET & ODD & NIP \\
\hline SHE & HID & HOD & LED & RAW & ORE \\
\hline TEN & TOY & LAX & LAY & $\mathrm{BAG}$ & PAW \\
\hline USE & RAW & RUT & DUE & BED & RUM \\
\hline WAS & RUG & SAC & SAT & ARC & SOD \\
\hline WIN & PIN & WRY & HIM & JOY & LAX \\
\hline
\end{tabular}

*High frequency words occurred with a frequency greater than 100 times per million; medium-frequency words occurred with a frequency between 38 and 50 times per million; and lowfrequency words occurred with a frequency of less than 5 times per million.

tThese lists were obtained from McIntire (1963).

tThe present authors were unable to locate this word in the Thorndike and Lorge word source. 
Because both of the two analyses described above indicated that the PA lists were not associated with any significant differential effects of the trials to criterion measure, the PA lists from the two word sources were combined and treated as a single variable for the next data analysis that was performed. A two-way analysis of variance revealed no significant difference between the entire PA list derived from the Thorndike-Lorge source and the entire list derived from the Kucera-Francis source $(F=1.39$; $\mathrm{df}=1 / 84)$, no significant difference between reinforcement conditions $(\mathrm{F}=2.12 ; \mathrm{df}=2 / 84)$, and no significant interaction of these factors $(F=2.36$; $\mathrm{df}=2 / 84$ ) at the .05 probability level. None of the results obtained give support to the hypothesis that a higher frequency word can act as a reinforcer in the learning of a PA list comprised of lower frequency words, as measured by word count sources. Consequently, the present results fail to replicate McIntire's original data.

It must be emphasized, however, that Premack's principle of reinforcement has not been adequately tested in either this or McIntire's experiment, since response probabilities were not measured in either experiment in the free choice situation required by the Premack reinforcement principle. For an acceptable test of the Premack hypothesis to be made, the following minimum conditions must be met: (a) all responses that are to be employed in the reinforcement contingency must be made concurrently available to $S$, so that $S$ is free to choose between them; (b) having established the probabilities of all responses freely and concurrently available to $S$, the more probable response must either be made contingent upon a less probable response, or it must occur subsequent to, and in close temporal proximity with the less probable responses; (c) subsequent to the completion of the experimental conditions described in (b), the less probable response must then be made contingent upon the more probable response. Only if reinforcement occurs in the conditions described under (b), and fails to occur under the condition described in (c), is support obtained for the Premack principle.
Regarding the possible application of the Premack principle to the acquisition of PA lists, the minimum empirical procedures required translate operationally to the following. Having established the base rates of occurrence of spoken words for an individual S, lists of stimulus words and response words of equal probabilities would have to be constructed, as would lists of reinforcement words of greater and of lesser probabilities than the stimulus words and the response words. By using the standard PA anticipation method for learning PA lists, and following the response word with either a high or low probability reinforcement word, depending upon the experimental condition being tested, it could be determined whether the reinforcement outcome predicted by the Premack principle would indeed be obtained. Any purported experimental tests of the Premack principle that employ conditions other than those just described must necessarily be inadequate. On the other hand, the probability of an experiment of this type being conducted must necessarily be small, since the procedures required to establish base rates of occurrence of individual verbalizations are obviously expensive and time consuming.

\section{REFERENCES}

Hawker, J. The influence of training procedure and other task variables in paired-associate learning. Journal of Verbal Learning \& Verbal Behavior, 1964, 3, 70-76.

Kausler, D., McLaughlin, B., \& Kulik, J. S-R and R-S paired-associate learning with varying occurrence of response members. Journal of Verbal Learning \& Verbal Behavior, 1962, 1, 85-90.

Kucera, H., \& Francis, W. Computational analysis of present-day American English. Providence, R. I: Brown University Press, 1967

McIntire, R. Reinforcement and Verbal Learning: A test of the Premack hypothesis. Psychological Reports. 1963, 12. 99-102.

Premack, D. Toward empirical behavior laws: I. Positive reinforcement. Psychological Review, 1959, 66, 219-233.

Premack, D. R einforcement theory. In D. Levine (Ed.), Nebraska symposium on motivation. Lincoln: University of Nebraska Press, 1965, Pp. 123-180.

Thorndike, E., \& Lorge, I. The teacher's word book of 30,000 words. New York: Teacher's College, Columbia University, 1944.

(R eceived for publication July 29, 1974.) 